\title{
Range of Motion After Total Hip Arthroplasty: Experimental Verification of the Analytical Simulator*
}

\author{
B. Jaramaz ${ }^{1,2}$, C. Nikou ${ }^{2}$, D.A. Simon ${ }^{1,2}$, A.M. DiGioia III ${ }^{1,2}$ \\ branko@cs.cmu.edu,costa+@andrew.cmu.edu, das@ri.cmu.edu,digioia@cs.cmu.edu \\ ${ }^{1}$ Center for Orthopaedic Research \\ ${ }^{2}$ Robotics Institute \\ Shadyside Hospital \\ Pittsburgh, PA 15232 \\ Carnegie Mellon University \\ Pittsburgh, PA 15213
}

\begin{abstract}
Dislocation following total hip replacement surgery represents a significant cause of early failure, incurring additional medical costs. The causes of dislocation are multifactorial and are related to surgical approach, soft tissue tension, prosthetic design, and most important, orientation of components. This paper describes experimental verification of our analytical approach for predicting implant impingement and dislocation. Once fully developed and tested, this analytical methodology could be used as a preoperative simulation tool that will present surgeons with information about the "safe" range of motion and chance of dislocation based on selected component positions, allowing for the surgical plan to be optimized based on this criterion. Coupled with a computer-assisted clinical system for precise implant positioning, this approach could significantly reduce the postoperative risk of dislocation, maximize "safe" range of motion and minimize impingement.
\end{abstract}

Keywords: range of motion analysis, total hip replacement, computer simulation, experimental validation.

\section{Introduction}

The incidence of implant dislocation following total hip replacement surgery ranges between 2 and $6 \%[3,14]$ and represents a significant cause of early implant failure, incurring additional costs to the total surgery expenses. The causes of dislocation after total hip arthroplasty (THA) are related to factors such as: surgical approach, soft tissue tension, prosthetic design, and most importantly, orientation of components.

We have developed a preoperative analytical simulator that takes into account implant design, placement and orientation, and predicts the range of motion (ROM) and impingement limits. The simulator will enable surgeons to preoperatively optimize the choice of implant-related parameters in order to reduce the probability of implant impingement and dislocation. Coupled with CT-based three-dimensional preoperative planning and computer-guided positioning of implant components [4], this methodology has the potential of precise implementation, ensuring optimal outcomes with respect to risk of dislocation.

* This work was supported in part by a National Challenge grant from the National Science Foundation (award IRI-9422734) and by the Shadyside Hospital Competitive Research Fund. 
Based on the analytical methodology used for preoperative ROM simulation, and using an optical tracking system to record the position of components, we have developed an experimental procedure to verify the analytical results. The main goal of the experiment was to validate the analytical methodology, and hence enable clinical implementation of the ROM simulator. The simulator could then be used both preoperatively, as a part of the preoperative surgical planning procedure and intraoperatively, to measure the range of motion.

\section{Background}

The most common cause of dislocation after THA is implant impingement caused by malposition of components [14]. A number of researchers and clinicians have examined this phenomenon, in an effort to explain mechanisms of dislocation. Amstutz and Markolf [2] described three modes of dislocation. In the first mode, due to poor tissue tension, the prosthetic head climbs the socket wall and slips over the rim of the socket, without the neck impinging on the rim of the socket. In the second mode, the neck impinges on the socket wall at extremes of flexion, extension and abduction and levers the head from the socket. The third mode of dislocation is impingement of the neck on a bony prominence, which occurs most often in hyperextension.

Some researchers have tried to identify the range of cup orientations that are less prone to dislocations, based on the geometric similarity of human anatomies. Commonly accepted "safe" orientation of the cup is approximately $15^{\circ}$ of anteversion and $45^{\circ}$ of abduction (although it is dependent on the surgical approach). Lewinnek et al. [11] demonstrated that the cases falling in the zone of $15 \pm 10$ degrees of anteversion and $40 \pm 10$ degrees of abduction have an instability rate of $1.5 \%$, compared with a $6 \%$ instability rate for the cases falling outside this zone. The study took note of the surgical approach taken in each case. No attention was paid, however, to the cup design, head-toneck ratio of the femoral component and femoral component orientation. It is also important to realize that, because of variations in individual anatomies, there cannot be a single optimal orientation of hip replacement components.

The pelvis can assume different positions and orientations depending or whether an individual is lying supine (as during a CT-scan or routine X-rays), in the lateral decubitis position (as during surgery) or in critical positions during activities of normal daily living (like bending over to tie shoes or during normal gait). Definition of a "neutral" relative position of the pelvis and leg will significantly influence the measured amount of motion permitted before impingement and dislocation occurs. Therefore, it is necessary to uniquely define both the neutral orientation of the femur relative to the pelvis for all relevant positions and activities, and the relations between the femur with respect to the pelvis of the patient during each segment of leg motion. In current practice, researchers and clinicians measure and record relative joint motion of the hip joint using physiological terms to describe position of the leg in relation to somewhat loosely defined global and local body coordinate systems $[5,6]$. Less often, the range of motion measurements are performed in the context of selected daily living activities, such as tying shoes with one foot on the floor, climbing stairs, etc. [8]. 
According to McCollum et al. [14], a comparison of THAs as reported in orthopaedic literature reveals a much higher incidence of dislocation in patients who had THAs with a posterolateral approach. They showed that when the patient is placed in the lateral position for a posterolateral THA approach, the lumbar lordotic curve is flattened and the pelvis may be flexed as much as $35^{\circ}$. If the cup was oriented at $15^{\circ}-20^{\circ}$ of flexion with respect to the longitudinal axis of the body, when the patient stood up and the postoperative lumbar lordosis was regained, the cup was retroverted as much as $10^{\circ}-15^{\circ}$.

Although the mechanism of implant impingement is well understood, the attempts to model the phenomenon are limited mainly to experimental procedures, in which a physical model is created to simulate the range of motion of the femur with respect to the pelvis. Most investigators [1,14] realized that the head-to-neck ratio of the femoral component is the key factor of the implant impingement. However, few have attempted to quantify the relationship between the implant design and orientation and the incidence of dislocation. Some experimental studies examined how specific implant design influences the prosthetic range of motion. Amstutz et al. [1] examined experimentally the influence of different prosthetic designs and the influence of prosthetic orientation. Krushell et al. [9] used a similar setup to experimentally confirm the effects of the headneck ratio to likeliness of impingement and warned of the negative impact that certain long and extra long neck designs of modular implants can have on the range of motion.

Several researchers have examined the effect of acetabular cup design. Krushell et al. [10] evaluated the ROM of two types of elevated-rim liners compared with standard liners. They concluded that an optimally oriented elevated-rim liner may improve the joint stability with respect to implant impingement. Cobb et al. [3] have demonstrated statistically significant reduction of dislocations in the case of elevated-rim liners, compared to standard liners. The two-year probability of dislocation was $2.19 \%$ for the elevated liner, compared with $3.85 \%$ for standard liner. They raised the concern, however, of possible long-term effects of the elevated liner on wear and loosening. Initial results of a finite element study by Maxian et al. [12] indicate that the contact stresses and therefore the polyethylene wear are not significantly increased in the extended lip case.

Analytical modeling of range of motion has only recently become a subject of interest for researchers. Maxian et al. [13] have looked at the dislocation propensity for different liner designs. They used three-dimensional finite element models to evaluate points of impingement and subsequent angles of dislocation for different liner designs. They did not, however, consider dislocation in the context of range of motion. Jaramaz et al. [7] developed an analytical model for calculating range of motion for a given size and orientation of implant components. The model can successfully simulate the prosthetic impingement as a limiting point for any combination of physiological leg motion. In the current stage, the simulation is limited to a case when both the cup liner and the neck of the femoral implant are axisymmetric. In this work we present the experimental verification of that simulation model. 


\section{Methodology}

\subsection{Analytical modeling}

The parameters necessary to evaluate the prosthetic range of motion (PROM) limited by the neck-liner impingement are the head-neck ratio of the implant, the position of the acetabular cup and the relative position of the femoral implant with respect to the cup. The center of rotation of the hip joint coincides with the center of the head of the femoral implant. The angle $\theta$ between the axis of symmetry $\mathrm{Z}$ of the acetabular cup and the line of impingement OB defines the allowable angle of motion (Fig. 1). The limits of impingement create a cone within which the axis of the femoral neck (line OA) can move without impingement. The position of the neck axis with respect to the cone can be evaluated by observing its intersection with the plane P (Fig. 1), placed at an arbitrary distance normal to the $\mathrm{Z}$ axis; the cross section of the cone defines the impingement circle (if both the liner and the neck are axisymmetric), and the path of the axis of the femoral neck defines a curve in that plane. An example movement is shown in Fig. 1 in which the axis of the femoral neck begins at point $A$ and moves to point $B$ along the path $\mathrm{AB}$. The motion of the femoral neck can be derived from (and expressed as a function of) the physiological movement of the leg, described in terms of combined flexion, extension, abduction, adduction, and external and internal rotation. Evaluating the impingement in the plane as a function of leg movement reduces the analysis of the problem to a 2D space, greatly simplifying it, and allows for creation of a user-friendly computer interface. With this interface, the effects of implant design, or reorientation of components can be immediately visualized and parameters can be modified interactively until the optimal selection, position and orientation of components is found.

Fig. 2 shows an example of ROM simulation for two different cup orientations and for two identical sets of ROM exercises: (I) $90^{\circ}$ flexion $+15^{\circ}$ adduction + maximum internal rotation and (II) $10^{\circ}$ extension + maximum external rotation. As a result of reorienting the cup from $45^{\circ}$ abduction $+15^{\circ}$ flexion (Fig. 2a) to $50^{\circ}$ abduction $+5^{\circ}$ flexion (Fig. 2b), maximum internal rotation is reduced from $15.7^{\circ}$ to $4.3^{\circ}$ in the first exercise and maximum external rotation is increased from $45.8^{\circ}$ to $55.8^{\circ}$ in the second one.

\subsection{Experimental verification}

Experimental validation is a necessary step in order to make clinical use of the described analytical PROM simulation model. Although an existing body of experimental

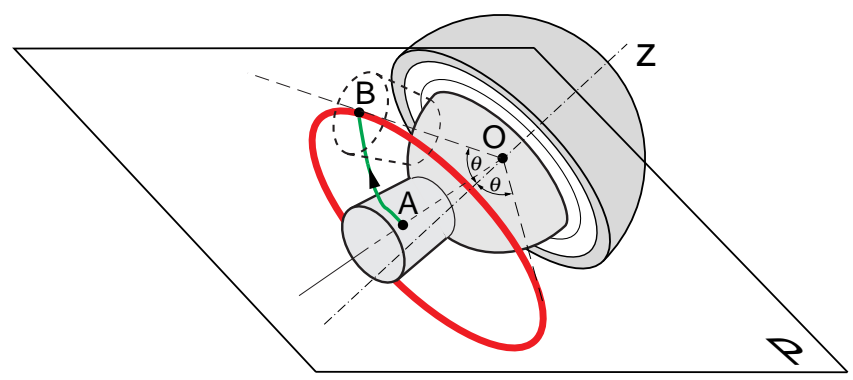

Figure 1. Implant components and the impingement limits 
research work on implant impingement and dislocation exists $[1,9,10,11]$, none of the available data can be used to fully verify our simulator, since no references are given about the neck size and the orientation of the femoral neck axis for any of the experiments. In this experiment we kept track of all the relevant data and compared the input and output data of the experiment with those coming from the analytical simulator. The experiment was performed using sawbones with the implanted total hip components. The position and orientation of all the components were measured and monitored using the Optotrak ${ }^{\mathrm{TM}}$ optical tracking system (Northern Digital, Inc.). The goal was to track the position of both the pelvis and the femur using rigidly attached optical targets during the range-of-motion exercises. Using measured orientations of the cup liner and the femoral neck axis with respect to the coordinate systems of the pelvis and the femur, the orientation of the neck axis with respect to the liner axis could be calculated at every instance of motion, and the results of the experiment could be visualized in the same way that is used for the analytical simulation.

\subsubsection{Registration}

For the registration stage of the PROM experiment, software was written and used to establish frames of reference for the pelvis, the femur, the acetabular cup implant, and the femoral implant, as well as for verification of these established frames. The registration process is described as a series of steps (Fig. 3). Before any of these steps are taken, however, the acetabular implant and the femoral implant (with a detachable head) must be firmly and properly affixed to the pelvis and femur, respectively:

1. Pointer Calibration - A pointer attached to an Optotrak ${ }^{\mathrm{TM}}$ LED tracker is calibrated. This process establishes a transformation between the origin of the LED tracker's coordinate system and the tip of the pointer. Calibration is performed by pivoting the pointer tip about a fixed point. The pivot point may either be fixed relative to the camera, or relative to a second LED tracker. After calibration, the pointer can be used to determine the location of various 3D points in order to establish pelvis and femoral frames of reference (FOR), as explained below.

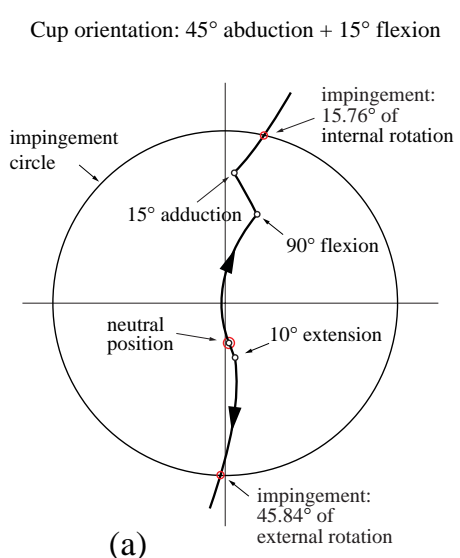

(a)

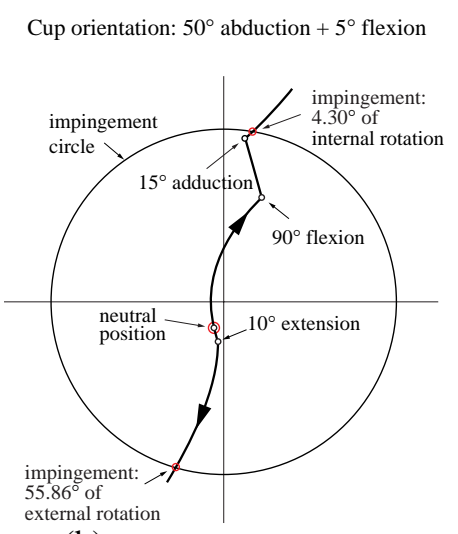

(b)

Figure 2. Example of simulator output for two cup orientations 
2. Pelvic Registration (Fig. 3a) - an LED tracker is attached to the pelvis and used to establish a pelvic FOR. We assume that the pelvic coordinate system has one (x) coordinate axis going through the centers of the acetabula and the other two in the perpendicular plane $\mathrm{P}_{1}$ which is also the symmetry plane of the pelvis. We define a plane $\mathrm{P}_{2}$ so that it touches the pubic tubercle and the anterior superior iliac spine. The second $(y)$ coordinate axis is then defined as the intersection of the plane $\mathrm{P}_{1}$ with the plane parallel to $\mathrm{P}_{2}$ that contains the first coordinate axis; the third $(\mathrm{z})$ coordinate axis is perpendicular to the first two. We place the pelvis on the Pelvic Calibration Plate in order to establish the coordinate system of the pelvis. The Pelvic Calibration Plate is an aluminum plate with two coordinate axes etched on its surface. The pelvis is placed on the plate at the position that corresponds to the definition of the plane $\mathrm{P}_{2}$. Using the pointer, we collect points on the plate that correspond to the pelvic origin, $\mathrm{X}$-axis, and $\mathrm{Y}$-axis relative to the tracker attached to the pelvis. With these points, we can establish a homogenous transformation ${ }^{\text {pelopto }} \mathbf{T}_{\text {pelvis }}$ which describes the pelvic frame relative to the LED tracker on the pelvis, as well as its inverse ${ }^{\text {pelvis }} \mathbf{T}_{\text {pelopto. }}$.

3. Cup Adapter Calibration - We collect two more points, this time relative to a LED tracker attached to the Cup Adapter. The Cup Adapter is designed such that these two points establish the cup axis vector when the Cup Adapter is properly placed in the acetabular cup implant. One of these points is placed so that when the Cup Adapter is seated in the cup, it indicates the centroid of the cup.

4. Cup Axis Registration (Fig. 3b) - With the positions of two Cup Adapter points known in the Cup Adapter tracker space, we can use the previously collected information ( ${ }^{\text {pelvis }} \mathbf{T}_{\text {pelopto }}$ and the Optotrak ${ }^{\mathrm{TM}}$-derived ${ }^{\text {pelopto }} \mathbf{T}_{\text {cupadapter }}$ ) to determine the cup axis vector in pelvic space. This vector can be used to establish a set of rotations (abduction, then flexion) that describe the cup implant's placement in the pelvis. Also collected is the center of pelvic rotation relative to the Pelvic tracker FOR ( $\left.{ }^{\text {pelopto }} \mathbf{P}_{\mathrm{cpr}}\right)$.

5. Femoral Registration (Fig. 3c) - Just like Pelvic Registration, a calibration plate is used to establish a femoral FOR relative to another LED tracker mounted to the femur. The femur is placed on the calibration plate so that the projections of both the center of the femoral head and the center of the femoral condyles fall in one of the coordinate axes on the plate. The homogenous transformations ${ }^{\text {femopto }} \mathbf{T}_{\text {femur }}$ and femur $\mathbf{T}_{\text {femopto }}$ can then be established.

6. Neck Adapter Calibration - The pointer is then used to collect points on the Neck Adapter, which is mounted to a separate LED tracker. When placed on the femoral implant's neck, these points define the neck axis vector relative to the femoral tracker.

7. Neck Axis Registration (Fig. 3d) - After the points on the Neck Adapter are calibrated, we place the neck adapter on the neck of the femoral implant. Since we know the neck axis vector position relative to the Neck Adapter tracker, and via Optotrak ${ }^{\mathrm{TM}}$ the position of the Neck Adapter relative to the Femoral tracker is also known, we can register the neck axis vector relative to the Femoral tracker (and by femur $\mathbf{T}_{\text {femopto }}$, relative to the Femoral FOR). This vector can be used to determine the placement (in terms of abduction, then anteversion) of the femoral implant relative to the femoral coordinate frame. 
8. Ball Target Calibration - The Ball Target Adapter, again attached to an LED tracker, can be calibrated so that it indicates, when placed on a sphere, the center of that sphere. This calibration gives the center of the sphere relative to the tracker attached to the Ball Target Adapter.

9. Registration of the Center of Femoral Rotation - The Ball Target Adapter is then placed on the head of the implant. We acquire the position of the head's center of rotation relative to both the Ball Target Adapter and, ultimately, to the Femoral tracker.

10. Verification of the Center of Rotation - Because we know femopto ${ }^{\text {cfr }}$ - the center of femoral rotation in the Femoral tracker's frame - using Optotrak ${ }^{\mathrm{TM}}$ and the previously collected information, we can determine the centroid of the femoral head(CFR) relative to the Pelvic tracker ( ${ }^{\text {pelopto }} \mathbf{P}_{\text {cfr }}$ ). When the head of the femoral implant is placed in the cup liner, the centroids of the cup (CPR) and the head (CFR) should coincide. This is verified by: ${ }^{\text {pelopto }} \mathbf{T}_{\text {femopto }} *$ femopto $\mathbf{P}_{\text {cfr }}={ }^{\text {pelopto }} \mathbf{P}_{\text {cfr }}$ When perfectly calibrated and executed, the distance from ${ }^{\text {pelopto }} \mathbf{P}_{\text {cpr }}$ to ${ }^{\text {pelopto }} \mathbf{P}_{\text {cfr }}$ should be zero.

\subsubsection{Experiment}

The final step of the experiment is shown in Fig. 3e. The pelvis, with its LED tracker still attached, was mounted in position above the laboratory table. The femur, also with a tracker, was held in location to the pelvis with elastic bands to give freedom of rotation

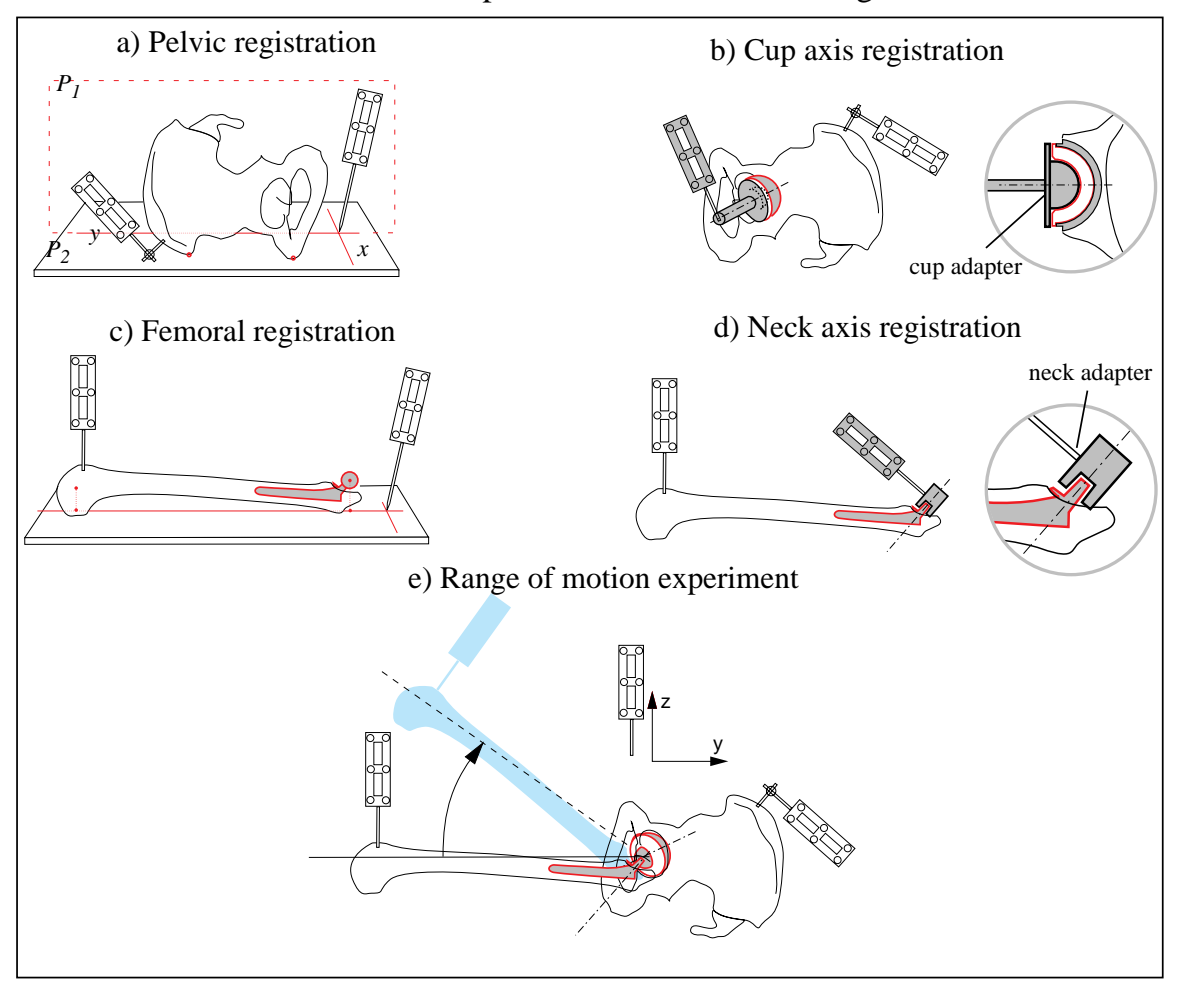

Figure 3. Five steps of the experiment 
but to keep the femoral implant from dislocating prematurely (before an impingement). The software used both the fixed, stored transformations that were determined during registration and the transformation (namely ${ }^{\text {pelopto }} \mathbf{T}_{\text {femopto }}$ ) that was constantly updated via Optotrak ${ }^{\mathrm{TM}}$. These transformations determined both a proper neutral position (when the femoral and pelvic coordinate orientations were aligned) and the current point on the impingement graph (by determining the neck axis vector in the cup's coordinate system). The software will plot, when specified by a foot pedal press, points on the impingement graph similar to that created by the analytical simulator (Fig. 5).

\section{Results}

The implant components used in the experiment were the HGP II acetabular cup and the Centralign femoral stem with a $28 \mathrm{~mm}$ head and short neck length (both manufactured by Zimmer, Inc.). The cup liner and the femoral implant neck are both axisymmetric. The components were cemented in the bone phantoms; the femoral component was placed so that the center of the femoral head remains close to the original head center of the uncut femur, and the acetabular component is placed so that the outer face is flush with the acetabular rim.The orientation of the components with respect to bone coordinate systems, as described in the previous section, were:

- Neck: 41.2 degrees of abduction and 17.6 degrees of anteversion, and

- Cup: 50.4 degrees of abduction followed by 2.6 degrees of flexion.

The error in center of rotation (the distance between the measured centers of the femoral head and the center of the liner) during the experiment was $0.87 \mathrm{~mm}$. Center of rotation errors for previous calibration runs ranged from $0.18 \mathrm{~mm}$ to $2 \mathrm{~mm}$.

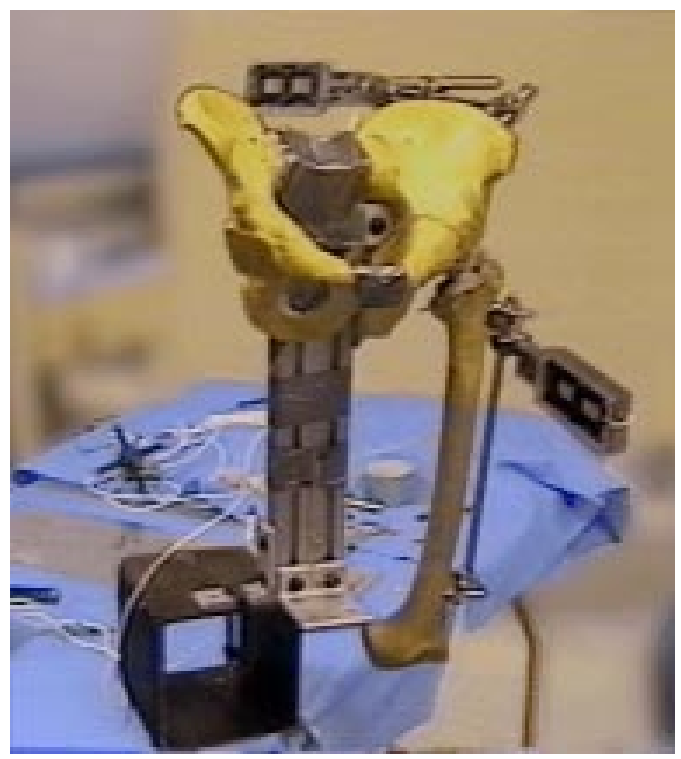

Figure 4. Experimental setup 


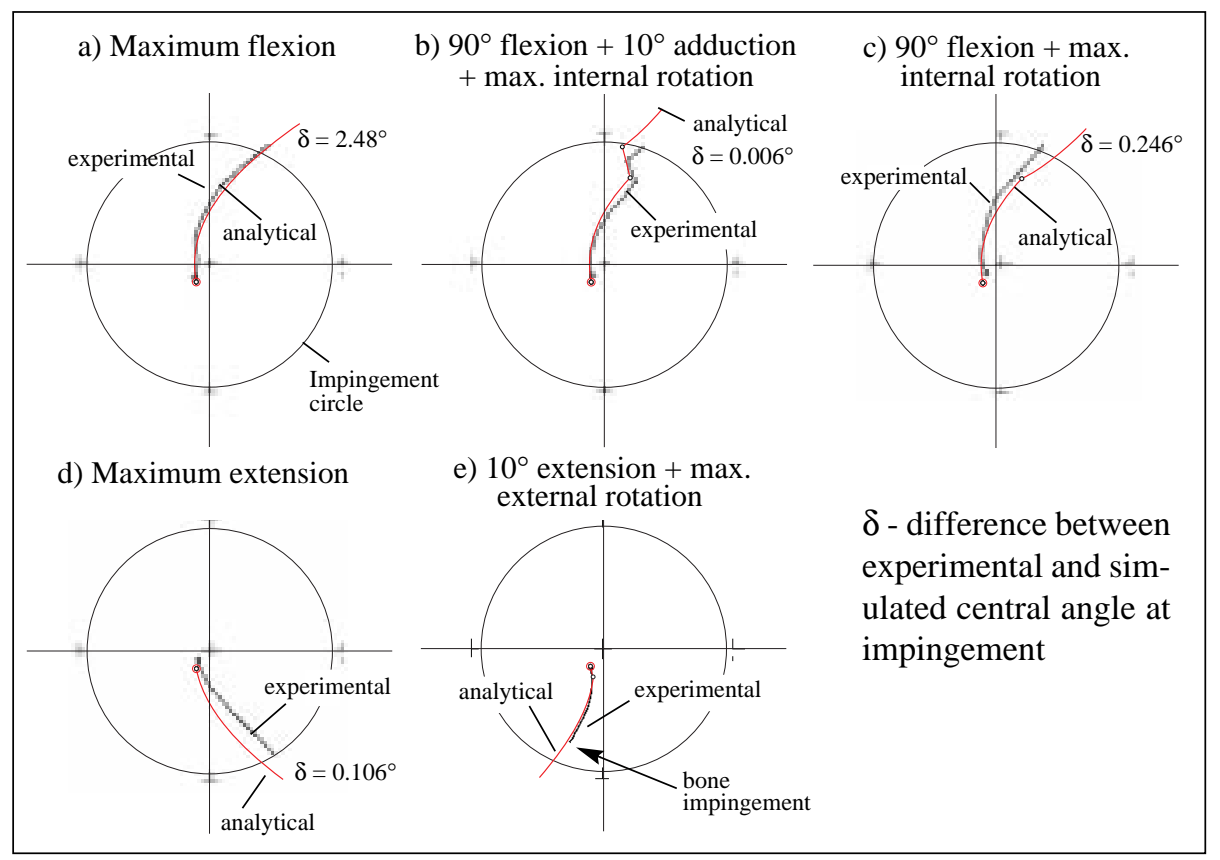

Figure 5. Analytical and experimental results of range of motion tests

The pelvis was firmly attached to an aluminum post, allowing full motion of the femur, and reorientation to make all the targets visible for every exercise (Fig. 4). Five range of motion exercises were performed. The motion sets were:

- Maximum flexion in neutral abduction/adduction (Fig. 5a) - The femur is flexed until impingement occurs.

- Maximum internal rotation after 90 degrees of flexion and 10 degrees adduction (Fig. 5b)

- Maximum internal rotation after 90 degrees flexion in neutral abduction/adduction (Fig. 5c)

- Maximum extension (Fig. 5d) - The femur is extended until impingement occurs.

- Maximum external rotation after 10 degrees of extension (Fig. 5e).

All trajectories started from near neutral position (within an error of \pm 2 degrees) and followed the movement set until impingement - either implant or bone - was observed. The only motion for which bony impingement was observed was external rotation after 10 degrees of extension. The difference angle $\delta$, between the central angle of the experimentally detected impingement and the central angle of impingement predicted by the simulation, varied from $0.006^{\circ}$ to $2.48^{\circ}$. The large value of $\delta$ during the maximum flexion exercise is not the result of a calculation error but rather the consequence of a difficulty in experimental detection of the impingement point. 


\section{Discussion}

We compared analytical simulation of ROM until impingement with those measured in the experimental setup. Experimentally detected values of impingement show that the simulated values are correctly calculated in all cases except one, in which bony impingement occurred prior to implant impingement. Femoral motion was generated manually without the use of special guides. Motions were performed by first visually assessing the direction of motion relative to the pelvis, and then rehearsing the key positions. Attempt was made to mimic the exact motions specified by the simulator. The recorded motion paths closely approximate the simulated values.

Proposed research addresses the clinically relevant issue of implant dislocation following total hip replacement (THR). The ROM simulator can predict implant impingement and dislocation on a patient-specific basis, based on a patient's CT-scan data, and the selected design and orientation of the implant. The results predicted by the simulator were verified experimentally. This work is a step toward future clinical use of this analytical simulation coupled with the clinical navigational system for hip implant positioning (HipNav). By coupling realistic biomechanical and kinematic preoperative planning with the precise execution we expect to take full advantage of this image-guided system.

\section{References}

[1] Amstutz H.C., et al.: Range of Motion Studies for Total Hip Replacements. Clinical Orthopaedics and Related Research (111), September 1975, 124-130.

[2] Amstutz, H.C. and Markolf, K.L.: Design features in total hip replacement. In Harris W.H. (ed.): Proceeding s of the Second Open Scientific Meeting of the Hip Society, New York, C.V.Mosby, 1974.

[3] Cobb T.K., Morrey B.F., Ilstrup D.M.: The elevated-rim acetabular liner in total hip arthroplasty: Relationship to postoperative dislocation. JBJS, Vol 78-A, N0. 1, January 1996, 80-86.

[4] DiGioia A.M., et al.: HipNav: Preoperative planning and intraoperative navigational guidance for acetabular implant placement in total hip replacement surgery. 2nd CAOS, Univ. of Bern, Switzerland, 1996.

[5] Gerhardt J.J. and Ripstein J.: Measuring and Recording of Joint Motion, Instrumentation and Techniques. Hogrefe \& Huber Publishers, Toronto, 1990.

[6] Greene W.B. and Hecknman J.D.: The clinical measurement of joint motion. American Academy of Orthopaedic Surgeons, Rosemont, Illinois, 97-114, 1994.

[7] Jaramaz B., et al.: Simulation of implant impingement and dislocation in total hip replacement. CAR '96, 10th international symposium and exhibition, Paris, June 26-29, 1996.

[8] Johnston, R.C. and Smidt, G.L.: Hip motion measurement for selected activities of daily living. Clin. Orthop. Sept-Oct 1970; (72): 205-215.

[9] Krushell, R.J., Burke D.W., and Harris W.H.: Range of motion in contemporary total hip arthroplasty (the impact of modular head-neck components). The Journal of Arthroplasty Vol. 6 (2 1991): 97-101.

[10] Krushell, R.J., Burke D.W., and Harris W.H.: Elevated-rim acetabular components: Effect on range of motion and stability in total hip arthroplasty. The Journal of Arthroplasty 6, Suppl. October 1991: 1-6.

[11] Lewinnek G.E., et al.: Dislocation after total hip-replacement arthroplasties. JBJS: 217-220, Vol. 60-A, No.2, March 1978.

[12] Maxian T.A., et al.: Femoral head containment in total hip arthroplasty: Standard vs. extended lip liners. 42nd Annual meeting, Orthopaedic Research society, February 19-22, 1966, Atlanta, Georgia, 421.

[13] Maxian T.A., et al.: Finite element modeling of dislocation propensity in total hip arthroplasty. 42nd Annual meeting, Orthopaedic Research society, February 19-22, 1966, Atlanta, Georgia, 259-44.

[14] McCollum, D.E. and W.J. Gray. Dislocation after total hip arthroplasty (causes and prevention). Clinical Orthopaedics and Related Research 261 (1990): 159-170. 